\title{
Inhibitory effect of high protein intake on nephrocalcinogenesis in female rats
}

\author{
BY J. G. H. STERCK ${ }^{1,2}$, J. RITSKES-HOITING A2 AND A. C. BEYNEN ${ }^{1,2 *}$ \\ ${ }^{1}$ Department of Human Nutrition, Agricultural University, PO Box 8129, 6700 EV Wageningen \\ and 'Department of Laboratory Animal Science, State University, PO Box 80.166, 3508 TD \\ Utrecht, The Netherlands
}

(Received 25 March 1991-Accepted 7 May 1991)

\begin{abstract}
Increased intakes of protein have been shown to reduce kidney calcification (nephrocalcinosis) in female rats. Two questions were addressed in the present study. First, can protein-induced inhibition of nephrocalcinosis be demonstrated when the diets used are balanced for calcium, magnesium and phosphorus in the added protein? Second, can the protein effect be explained by the frequently observed magnesiuria after giving high-protein diets? Nephrocalcinosis was induced in female rats by giving purified diets containing $151 \mathrm{~g}$ casein/ $/ \mathrm{kg}$ and either an increased concentration of $P(6 \mathrm{v} .2 \mathrm{~g} / \mathrm{kg})$ or a decreased concentration of $\mathrm{Mg}(0.1 v .0 .4 \mathrm{~g} / \mathrm{kg})$. To these diets $151 \mathrm{~g} \mathrm{ovalbumin} / \mathrm{kg}$ was added at the expense of glucose, and the diets were balanced for $\mathrm{Ca}, \mathrm{Mg}$ and $\mathrm{P}$ in ovalbumin. The diets were given for $29 \mathrm{~d}$. In rats fed on the diet containing $151 \mathrm{~g}$ protein $/ \mathrm{kg}$, an increased intake of $P$ or a decreased intake of $\mathrm{Mg}$ caused nephrocalcinosis as measured chemically by analysis of kidney $\mathrm{Ca}$ as well as histologically by scoring kidney sections stained according to Von Kossa's method. The addition of ovalbumin to the diet prevented the induction of nephrocalcinosis. High P intake and low Mg intake with the low-protein diets induced enhanced loss of albumin in urine, suggesting that nephrocalcinosis caused kidney damage. Increased protein intake with a non-calcinogenic diet also caused increased albumin excretion in urinc. Irrespective of the composition of the background diet, increased protein intake caused increased urinary excretion of $\mathrm{Mg}$. When all dietary groups were considered, differences in nephrocalcinosis and urinary $\mathrm{Mg}$ output were not proportionally related.
\end{abstract}

Nephrocalcinosis: Phosphorus: Magnesium: Protein: Rat

Nephrocalcinosis refers to the deposition of calcium phosphates in the corticomedullary region of the kidney. In general, the primary lesion is an intratubular formation of calcium phosphate precipitates. Diets with either high concentrations of phosphorus or low concentrations of magnesium are known to promote nephrocalcinosis in the rat (Hellerstein et al. 1957; Bunce et al. 1965; Du Bruyn, 1972; Parker, 1985; Hoek et al. 1988; Mars et al. 1988). Decreasing the intake of $\mathrm{P}$ or increasing that of $\mathrm{Mg}$ not only prevents nephrocalcinosis but also causes magnesiuria (Goulding \& Malthus, 1969; Bunce \& Bloomer, 1972; Draper et al. 1972; Hitchman et al. 1979; Harwood, 1982; Al-Modhefer et al. 1986). Possibly there is a relationship between urinary $\mathrm{Mg}$ excretion and nephrocalcinogenesis. This is supported by in vitro observations that $\mathrm{Mg}$ inhibits the precipitation of calcium phosphates (Boulet et al. 1962).

High-protein diets have been shown to induce magnesiuria (Schneider \& Menden, 1988). Thus, it could be suggested that diets rich in protein inhibit nephrocalcinogenesis. Indeed, evidence has been reported that high protein intakes reduce the degree of nephrocalcinosis in rats (Eklund et al. 1973; Schneider \& Menden, 1988). However, the protein preparations

$$
\text { * For reprints. }
$$


used in these studies were not pure, and the experimental diets were not balanced for components other than protein in the protein preparations, such as $\mathrm{Ca}, \mathrm{Mg}$ or $\mathrm{P}$. This prompted us to re-address the question whether the amount of protein in the diet affects nephrocalcinosis in female rats, or rather whether high protein intakes can counteract nephrocalcinogenesis induced by either high $\mathrm{P}$ or low $\mathrm{Mg}$ concentrations in the diet. In addition, we tested our hypothesis that protein effects on nephrocalcinosis are related to protein-induced magnesiuria.

\section{MATERIALS AND METHODS}

Animals, housing and diets

Two experiments of the same design were performed. The interval between the two experiments was about 12 weeks. Outbred, female Wistar (Cpb: WU) rats, aged 7-8 weeks, were used throughout. They had been fed on a commercial pelleted diet (RMHB; Hope Farms, Woerden). All rats received a purified, pre-experimental diet and demineralized water ad lib. The pre-experimental diet contained $(\mathrm{g} / \mathrm{kg}): 151$ protein $0.4, \mathrm{Mg}$ and $2 \mathrm{P}$ (Table 1). The pre-experimental period was inserted before the experimental period in order to eliminate possible effects of the commercial diet and to allow the rats to adjust to a purified diet. After the pre-experimental period of $10 \mathrm{~d}$ (day 0 ), the rats were divided into four groups of either six (Expt 1) or eight rats each (Expt 2). The groups were formed so that their body-weight distributions were similar. Expt 1 was performed to see whether high protein intake counteracts P-induced nephrocalcinosis. In Expt 1, one group remained on the pre-experimental diet and the other three groups were switched to purified diets containing $0.4 \mathrm{~g} \mathrm{Mg} / \mathrm{kg}$ and different amounts of $\mathrm{P}(2$ or $6 \mathrm{~g} / \mathrm{kg})$ and protein $(15 \mathrm{l}$ or $302 \mathrm{~g} / \mathrm{kg}$ ). Expt 2 was carried out to check, in part, the reproducibility of Expt 1 and to find out whether high protein intake counteracts nephrocalcinosis as induced by $\mathrm{Mg}$ restriction. In Expt 2, all groups were transferred from the pre-experimental diet to a diet containing $6 \mathrm{~g} \mathrm{P} / \mathrm{kg}$ and different amounts of $\mathrm{Mg}(0.4$ or $0.1 \mathrm{~g} / \mathrm{kg})$ and protein $(151$ or $302 \mathrm{~g} / \mathrm{kg})$. All diets contained $5 \mathrm{~g} \mathrm{Ca} / \mathrm{kg}$. The experimental period lasted $29 \mathrm{~d}$.

Table 1 shows the composition of the diets. Extra protein was added in the form of ovalbumin at the expense of glucose, and the diets were balanced for $\mathrm{Ca}, \mathrm{Mg}$ and $\mathrm{P}$ in the protein preparation. The calculated amounts of $\mathrm{Ca}, \mathrm{Mg}, \mathrm{P}$ and protein in the diets agreed well with the analysed concentrations (Table 1). All diets were in powdered form and stored at $4^{\circ}$ until used. Before feeding, the meal was mixed with demineralized water in the proportion $5: 1(\mathrm{w} / \mathrm{w})$. The animals were fed ad lib. and had free access to demineralized water. The compositions of the pre-experimental diet and the diets containing $6 \mathrm{~g} \mathrm{P} / \mathrm{kg}$ and $0.4 \mathrm{~g} \mathrm{Mg} / \mathrm{kg}$ were identical for Expts 1 and 2. Separate batches of diets were made for the two experiments.

During the experimental period (days 0-29) the rats were housed individually in metabolism cages which were located in a room with controlled temperature $\left(20-22^{\circ}\right)$, humidity (55-65\%) and lighting (12 h light-dark cycle with light from 06.00-18.00 hours).

\section{Collection of samples and chemical analyses}

From days 13 to 15 (period 1) and days 27 to 29 (period 2), urine and faeces from each rat were collected quantitatively. Excreta were collected daily and stored at $4^{\circ}$. After complete collection, part of the urine was acidified to $\mathrm{pH} \mathrm{1-2}$ with $6 \mathrm{M}$-hydrochloric acid and centrifuged $(1200 \mathrm{~g}, 10 \mathrm{~min})$. The supernatant fraction was frozen at $-20^{\circ}$ until analysed for $\mathrm{Ca}, \mathrm{Mg}$ and $\mathrm{P}$. Faeces were freeze-dried, homogenized and weighed. A sampie of $150 \mathrm{mg}$ was ashed at $500^{\circ}$ for $17 \mathrm{~h}$ and then dissolved in $6 \mathrm{M}-\mathrm{HCl}$. Feed samples were 
Table 1. Composition of diets $(\mathrm{g} / \mathrm{kg})$

\begin{tabular}{|c|c|c|c|c|c|c|}
\hline \multirow[b]{2}{*}{$\begin{array}{l}\text { Expt ... } \\
\text { Protein ... } \\
\text { Phosphorus ... } \\
\text { Magnesium ... } \\
\text { Ingredient }\end{array}$} & \multicolumn{6}{|c|}{ Diet* } \\
\hline & $\begin{array}{c}1 / 2 \dagger \\
151 \\
2 \\
0.4\end{array}$ & $\begin{array}{c}1 \\
302 \\
2 \\
0 \cdot 4\end{array}$ & $\begin{array}{c}1 / 2 \\
151 \\
6 \\
0 \cdot 4\end{array}$ & $\begin{array}{c}l / 2 \\
302 \\
6 \\
0 \cdot 4\end{array}$ & $\begin{array}{c}2 \\
151 \\
6 \\
0 \cdot 1\end{array}$ & $\begin{array}{c}2 \\
302 \\
6 \\
0 \cdot 1\end{array}$ \\
\hline $\begin{array}{l}\text { Ovalbumin } \ddagger \\
\text { Glucose } \\
\mathrm{CaCO}_{3} \\
\mathrm{NaH}_{2} \mathrm{PO}_{4}-2 \mathrm{H}_{2} \mathrm{O} \\
\mathrm{Na}_{2} \mathrm{CO}_{3} \\
\mathrm{MgCO}_{3} \\
\text { Constant components }\end{array}$ & $\begin{array}{r}709 \cdot 3 \\
12 \cdot 4 \\
5 \cdot 0 \\
10 \cdot 2 \\
1 \cdot 4 \\
261 \cdot 7\end{array}$ & $\begin{array}{c}151 \cdot 0 \\
559 \cdot 26 \\
12 \cdot 24 \\
4 \cdot 2 \\
10 \cdot 48 \\
1 \cdot 12 \\
261 \cdot 7\end{array}$ & 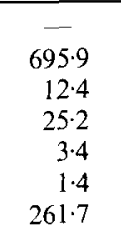 & $\begin{array}{c}151 \cdot 0 \\
545 \cdot 86 \\
12 \cdot 24 \\
24 \cdot 4 \\
3 \cdot 68 \\
1 \cdot 12 \\
261 \cdot 7\end{array}$ & $\begin{array}{l}-\bar{c} \\
696 \cdot 88 \\
12 \cdot 4 \\
25 \cdot 2 \\
3 \cdot 4 \\
0 \cdot 42 \\
261 \cdot 7\end{array}$ & $\begin{array}{l}151 \cdot 0 \\
546 \cdot 98 \\
12 \cdot 24 \\
24 \cdot 4 \\
3 \cdot 68 \\
-7 \\
261 \cdot 7\end{array}$ \\
\hline $\begin{array}{l}\text { Chemical analysis } \\
\text { Calcium } \\
\text { Magnesium } \\
\text { Phosphorus } \\
\text { Crude protein }\end{array}$ & $\begin{array}{l}4 \cdot 7 / 4 \cdot 0 \\
0 \cdot 4 / 0 \cdot 3 \\
2 \cdot 2 / 1 \cdot 8 \\
140 / 138\end{array}$ & $\begin{array}{l}4 \cdot 8 \\
0 \cdot 4 \\
2 \cdot 0 \\
260\end{array}$ & $\begin{array}{l}4 \cdot 7 / 4 \cdot 2 \\
0 \cdot 4 / 0 \cdot 3 \\
5 \cdot 6 / 5 \cdot 4 \\
139 / 134\end{array}$ & $\begin{array}{r}4 \cdot 7 / 4 \cdot 2 \\
0 \cdot 4 / 0 \cdot 4 \\
5 \cdot 7 / 5 \cdot 0 \\
260 / 254\end{array}$ & $\begin{array}{c}4 \cdot 2 \\
0 \cdot 1 \\
5 \cdot 0 \\
135\end{array}$ & $\begin{array}{l}4 \cdot 1 \\
0 \cdot 1 \\
4 \cdot 8 \\
260\end{array}$ \\
\hline
\end{tabular}

* Dietary variables refer to the calculated amounts of protein, $\mathrm{P}$ and $\mathrm{Mg}$.

$\uparrow$ Pre-experimental diet for both experiments.

$\ddagger$ Chemical analysis showed that the ovalbumin preparations contained ( $\mathrm{g} / \mathrm{kg}$ for Expts 1 and 2 respectively) $0.44,0.42 \mathrm{Ca} ; 0.54,0.80 \mathrm{Mg} ; 1 \cdot 06,0.98 \mathrm{P}$.

$\S$ Constant components consisted of $(\mathrm{g} / \mathrm{kg}$ diet) : casein 151 , maize oil 25 , coconut fat 25 , cellulose $30, \mathrm{KCl} 1$, $\mathrm{KHCO}_{3} 7 \cdot 7$, mineral premix 10 , vitamin premix 12 . The compositions of the mineral and vitamin premixes have been described by Hoek et al. (1988).

processed as faeces samples. $\mathrm{Ca}$ and $\mathrm{Mg}$ in urine, faeces and feed samples were determined by atomic absorption spectroscopy (Hoek et al. 1988; Mars et al. 1988). P was analysed according to Taussky \& Shorr (1953). Non-acidified urine samples were frozen at $-20^{\circ}$ until analysed for albumin, lysozyme, urea and creatinine. Albumin in urine was determined by radial immunodiffusion (Mancini et al. 1965). Lysozyme in urine was detected by the agar plate diffusion method of Osserman \& Lawlor (1966).

At the end of the experiment (day 29) between 09.00 and 13.00 hours, the non-starved rats were anaesthetized with diethyl ether, and blood was obtained by orbital puncture into heparinized tubes. The anaesthetized rats were killed by decapitation (Expt 1) or exposure to carbon dioxide (Expt 2). Different methods of euthanasia were used in the two experiments for practical reasons. The kidneys from each rat were collected and after capsules had been removed they were weighed. The right kidneys were used for chemical analysis of $\mathrm{Ca}, \mathrm{Mg}$ and $\mathrm{P}$ as described for faeces and feed samples. The left kidneys were fixed in formalin and stained by Von Kossa's method (Mallory, 1961). The severity of nephrocalcinosis was graded on a scale from 0 (no $\mathrm{Ca}$ deposits) to 3 (severe calcinosis). To aid in scoring, four reference slides were used. The kidneys were scored in random order by three persons who were blinded to treatment modality. The score of each rat was the average score of the three assessors.

Plasma and urinary urea were determined with a test combination (Urea UV, MA-KIT 10 ROCHE; Roche Diagnostics, Basel, Switzerland). Creatinine in plasma and urine was also determined with a test combination (UNI-KIT II ROCHE; Roche Diagnostics). Both analyses were performed with the use of a COBAS-BIO autoanalyser (Hoffman-La Roche BV, Mijdrecht). 


\section{Statistical analyses}

Analysis of variance was performed to disclose protein, $\mathrm{P}$ and $\mathrm{Mg}$ effects, and $\mathrm{P} \times$ protein and $\mathrm{Mg} \times$ protein interactions. Scores of nephrocalcinosis were not distributed normally, and the Kruskal-Wallis rank statistics test was used to compare group means.

\section{RESULTS}

\section{Growth and feed intake}

Rats fed on the high-protein $(302 \mathrm{~g} / \mathrm{kg}$ ) diets showed significantly lower feed intakes and lower rates of body-weight gain (Table 2). In Expt 2, the amount of protein in the diet did not significantly affect body-weight gain but did influence feed intake (Table 3 ). The discrepancy between the effect of high protein intake on body-weight gain in Expts 1 and 2 may relate to the fact that in Expt 1 the group mean initial body-weights of the low- and high-protein groups were different. The lower initial body-weight of the high-protein group may have caused an overestimation of the observed depressing effect of high protein intake on weight gain. P intake ( $2 v .6 \mathrm{~g} / \mathrm{kg}$ ) did not significantly affect feed intake or body-weight gain. Body-weight gain was significantly depressed in rats fed on the low-Mg diets, while their feed intake was only slightly reduced (Table 3 ).

\section{Effects of $P$ intake at two dietary protein levels}

An increase in $\mathrm{P}$ intake caused a decreased urinary output of $\mathrm{Ca}$ and $\mathrm{Mg}$ and an increase of faecal excretion of $\mathrm{Mg}$ (Table 2). In rats fed on the high-P diets, urinary and faecal excretion and retention of $\mathbf{P}$ were significantly increased when compared with rats fed on the low-P diets.

In rats fed on the low-protein $(151 \mathrm{~g} / \mathrm{kg})$ diets, an increase in dietary $\mathrm{P}$ level from 2 to $6 \mathrm{~g} / \mathrm{kg}$ induced increased kidney concentrations of $\mathrm{Ca}, \mathrm{P}$ and $\mathrm{Mg}$. In rats fed on the highprotein diets, an increased intake of $P$ did not influence mineral concentrations of kidney. In rats fed on the low-protein diet, but not in those fed on the high-protein diet, an increased intake of $\mathrm{P}$ caused an increase in severity of nephrocalcinosis as determined histologically.

In rats fed on the low-protein diets, an increased $P$ intake tended to stimulate urinary excretion of albumin. Creatinine clearance, urea and creatinine in urine and plasma were not influenced by the $\mathrm{P}$ content of the diet.

\section{Effects of $\mathrm{Mg}$ intake at two dietary protein levels}

A decrease in $\mathrm{Mg}$ intake reduced $\mathrm{Mg}$ excretion in urine and faeces, lowered urinary $\mathrm{Ca}$ excretion, but did not systematically influence $\mathrm{P}$ excretion.

In rats fed on the low-protein diets, a decrease in $\mathrm{Mg}$ intake resulted in a significant increase in kidney weight. When the diet contained $151 \mathrm{~g}$ protein $/ \mathrm{kg}$, a decrease in dietary $\mathrm{Mg}$ caused significantly increased concentrations in kidney of $\mathrm{Ca}, \mathrm{Mg}$ and $\mathrm{P}$ and caused increased incidence and severity of nephrocalcinosis as assessed histologically. This $\mathrm{Mg}$ effect was not seen with the high-protein diet.

In rats fed on the low-protein diet, a decrease in $\mathrm{Mg}$ intake tended to cause an increase in urinary output of albumin. This $\mathrm{Mg}$ effect was not seen in rats fed on the high-protein diet. Lowering the $\mathrm{Mg}$ content of the diet increased the urea content in plasma. Urinary creatinine was not significantly influenced by the amounts of $\mathrm{Mg}$ in the diet. Creatinine clearance tended to be increased by low Mg intake. 
Table 2. Expt 1. Effects of protein and phosphorus intakes on growth and feed intakes, mineral balance and kidney function in rats

(Means with pooled standard errors for six rats per dietary group)

\begin{tabular}{|c|c|c|c|c|c|c|}
\hline \multirow[b]{2}{*}{$\begin{array}{l}\text { Protein }(\mathrm{g} / \mathrm{kg}) \ldots \\
\text { P }(\mathrm{g} / \mathrm{kg}) \ldots \\
\text { Magnesium }(\mathrm{g} / \mathrm{kg}) \ldots\end{array}$} & \multicolumn{4}{|c|}{ Diet* } & \multirow[b]{2}{*}{ Pooled SE } & \multirow[b]{2}{*}{$\begin{array}{l}\text { Statistical } \\
\text { significance }\end{array}$} \\
\hline & $\begin{array}{c}151 \\
2 \\
0 \cdot 4\end{array}$ & $\begin{array}{c}302 \\
2 \\
0 \cdot 4\end{array}$ & $\begin{array}{c}151 \\
6 \\
0.4\end{array}$ & $\begin{array}{c}302 \\
6 \\
0 \cdot 4\end{array}$ & & \\
\hline \multicolumn{7}{|l|}{ Body-wt (g) } \\
\hline Initial & $191 \cdot 1$ & $186 \cdot 8$ & 193.6 & $178 \cdot 2$ & $5 \cdot 5$ & \\
\hline Final & $224 \cdot 7$ & $212 \cdot 4$ & $232 \cdot 4$ & $206 \cdot 8$ & $5 \cdot 2$ & \\
\hline Wt gain $(\mathrm{g} / \mathrm{d})$ & $1 \cdot 2$ & $0 \cdot 9$ & $1 \cdot 4$ & $1 \cdot 0$ & $0 \cdot 1$ & Prot \\
\hline Feed intake $(\mathrm{g} / \mathrm{d})$ & $16 \cdot 8$ & $15 \cdot 4$ & $17 \cdot 5$ & $15 \cdot 5$ & 0.3 & Prot \\
\hline \multicolumn{7}{|c|}{ Mineral balance, period $1(\mathrm{mg} / \mathrm{d})$} \\
\hline \multicolumn{7}{|c|}{ 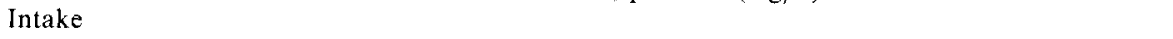 } \\
\hline Calcium & $73 \cdot 6$ & $70 \cdot 7$ & $74 \cdot 1$ & 66.9 & $2 \cdot 7$ & \\
\hline $\mathrm{Mg}$ & $6 \cdot 3$ & $5 \cdot 9$ & $6 \cdot 5$ & $5 \cdot 7$ & $0 \cdot 2$ & Prot \\
\hline $\mathrm{P}$ & $31 \cdot 3$ & $29 \cdot 5$ & $96 \cdot 7$ & $85 \cdot 4$ & $2 \cdot 7$ & Prot,P \\
\hline \multicolumn{7}{|l|}{ Urinary output } \\
\hline $\mathrm{Ca}$ & $0 \cdot 4$ & $1 \cdot 1$ & $0 \cdot 2$ & 0.3 & $0 \cdot 1$ & Prot,P,Prot $\times$ P \\
\hline $\mathrm{Mg}$ & 1.6 & 1.8 & 0.5 & 0.7 & $0 \cdot 1$ & $\mathrm{P}$ \\
\hline $\mathrm{P}$ & $3 \cdot 6$ & $3 \cdot 3$ & $40 \cdot 4$ & $35 \cdot 7$ & $1 \cdot 7$ & $\mathrm{P}$ \\
\hline \multicolumn{7}{|l|}{ Faecal output } \\
\hline $\mathrm{Ca}$ & $50 \cdot 1$ & $45 \cdot 1$ & $48 \cdot 3$ & $44 \cdot 4$ & $3 \cdot 2$ & \\
\hline $\mathrm{Mg}$ & $2 \cdot 7$ & $2 \cdot 2$ & $4 \cdot 0$ & $3 \cdot 3$ & $0 \cdot 2$ & Prot,P \\
\hline $\mathrm{P}$ & $15 \cdot 7$ & $14 \cdot 3$ & $29 \cdot 9$ & 23.8 & $1 \cdot 3$ & Prot,P \\
\hline \multicolumn{7}{|l|}{ Retention } \\
\hline $\mathrm{Ca}$ & $23 \cdot 1$ & $24 \cdot 5$ & $25 \cdot 6$ & $22 \cdot 2$ & $3 \cdot 5$ & \\
\hline $\mathrm{Mg}$ & $2 \cdot 0$ & $2 \cdot 0$ & $1 \cdot 8$ & $1 \cdot 7$ & $0 \cdot 2$ & \\
\hline$P$ & $12 \cdot 1$ & $11 \cdot 9$ & $26 \cdot 5$ & $26 \cdot 0$ & $2 \cdot 5$ & $\mathbf{P}$ \\
\hline \multicolumn{7}{|c|}{ Mineral balance, period $2(\mathrm{mg} / \mathrm{d})$} \\
\hline Intake & & & & & & \\
\hline $\mathrm{Ca}$ & $76 \cdot 2$ & $72 \cdot 3$ & $77 \cdot 2$ & $69 \cdot 3$ & $2 \cdot 1$ & Prot \\
\hline $\mathrm{Mg}$ & $6 \cdot 5$ & $6 \cdot 0$ & $6 \cdot 7$ & $5 \cdot 9$ & $0 \cdot 2$ & Prot \\
\hline $\mathrm{P}$ & $32 \cdot 4$ & $30 \cdot 2$ & $100 \cdot 7$ & $88 \cdot 5$ & $1 \cdot 7$ & Prot,P,Prot $\times P$ \\
\hline \multicolumn{7}{|l|}{ Urinary output } \\
\hline $\mathrm{Ca}$ & $0 \cdot 6$ & $1 \cdot 7$ & $0 \cdot 3$ & $0 \cdot 5$ & $0 \cdot 1$ & Prot,P,Prot $\times \mathrm{P}$ \\
\hline $\mathrm{Mg}$ & $1 \cdot 4$ & 1.9 & 0.6 & $0 \cdot 7$ & $0 \cdot 2$ & Prot,P \\
\hline $\mathrm{P}$ & $3 \cdot 1$ & $2 \cdot 9$ & $38 \cdot 9$ & $37 \cdot 6$ & $1 \cdot 3$ & $\mathrm{P}$ \\
\hline \multicolumn{7}{|l|}{ Faecal output } \\
\hline $\mathrm{Ca}$ & $53 \cdot 6$ & $49 \cdot 6$ & $52 \cdot 5$ & $45 \cdot 1$ & $2 \cdot 2$ & Prot \\
\hline $\mathrm{Mg}$ & $3 \cdot 0$ & $2 \cdot 3$ & $4 \cdot 3$ & $3 \cdot 7$ & $0 \cdot 2$ & Prot,P \\
\hline$P$ & $18 \cdot 6$ & $16 \cdot 1$ & $31 \cdot 5$ & $27 \cdot 3$ & $1 \cdot \overline{2}$ & Prot,P \\
\hline \multicolumn{7}{|l|}{ Retention } \\
\hline $\mathrm{Ca}$ & $22 \cdot 0$ & $21 \cdot 1$ & $24 \cdot 4$ & $23 \cdot 7$ & 1.9 & \\
\hline $\mathrm{Mg}$ & $2 \cdot 1$ & $1 \cdot 8$ & 1.9 & 1.5 & $0 \cdot 2$ & Prot \\
\hline $\mathrm{P}$ & $10 \cdot 8$ & $11 \cdot 2$ & $30 \cdot 3$ & $23 \cdot 6$ & $1 \cdot 1$ & Prot,P,Prot $\times \mathbf{P}$ \\
\hline \multicolumn{7}{|c|}{ Kidney variables } \\
\hline $\begin{array}{l}\text { Relative kidney wt } \\
(\mathrm{g} / \mathrm{kg} \text { body-wt) }\end{array}$ & $3 \cdot 4$ & $3 \cdot 8$ & $3 \cdot 6$ & $3 \cdot 9$ & $0 \cdot 2$ & Prot \\
\hline Dry wt (mg) & 182 & 190 & 195 & 190 & 6 & \\
\hline $\mathrm{Ca}(\mathrm{g} / \mathrm{kg})$ & $0 \cdot 36$ & 0.32 & $10 \cdot 42$ & $0 \cdot 20$ & $0 \cdot 2$ & Prot,P,Prot $\times$ P \\
\hline $\operatorname{Mg}(\mathrm{g} / \mathrm{kg})$ & $1 \cdot 03$ & $1 \cdot 02$ & $1 \cdot 21$ & 1.06 & 0.01 & $\mathbf{P}$ \\
\hline $\mathrm{P}(\mathrm{g} / \mathrm{kg})$ & $13 \cdot 0$ & 12.8 & $17 \cdot 3$ & $13 \cdot 4$ & $0 \cdot 1$ & $P$ \\
\hline \multicolumn{7}{|l|}{ Nephrocalcinosis } \\
\hline Incidence & $0 / 6$ & $0 / 6$ & $4 / 6$ & $0 / 6$ & & \\
\hline Severity & $0 \cdot 0^{\mathrm{a}}$ & $0 \cdot 0^{\mathrm{a}}$ & $2 \cdot 0^{\mathrm{b}}$ & $0 \cdot 0^{\mathrm{a}}$ & & \\
\hline
\end{tabular}


Table 2. (cont.)

\begin{tabular}{|c|c|c|c|c|c|c|}
\hline \multirow{4}{*}{$\begin{array}{l}\text { Protein }(\mathrm{g} / \mathrm{kg}) \ldots \\
\mathrm{P}(\mathrm{g} / \mathrm{kg}) \ldots \\
\text { Magnesium }(\mathrm{g} / \mathrm{kg}) \ldots\end{array}$} & \multicolumn{4}{|c|}{ Diet* } & \multirow[b]{4}{*}{ Pooled SE } & \multirow{4}{*}{$\begin{array}{l}\text { Statistical } \\
\text { significance }{ }^{\dagger}\end{array}$} \\
\hline & 151 & 302 & 151 & 302 & & \\
\hline & 2 & 2 & 6 & 6 & & \\
\hline & 0.4 & $0 \cdot 4$ & $0 \cdot 4$ & 0.4 & & \\
\hline \multicolumn{7}{|c|}{ Urinary output } \\
\hline Production $(\mathrm{ml} / \mathrm{d})$ & & & & & & \\
\hline Period: 1 & $11 \cdot 8$ & $12 \cdot 4$ & $9 \cdot 6$ & $11 \cdot 4$ & $4 \cdot 1$ & \\
\hline 2 & $11 \cdot 1$ & $14 \cdot 4$ & $9 \cdot 9$ & $12 \cdot 0$ & $5 \cdot 1$ & \\
\hline \multicolumn{7}{|l|}{$\mathrm{pH}$} \\
\hline Period: 1 & $8 \cdot 7$ & $8 \cdot 7$ & $7 \cdot 3$ & $7 \cdot 6$ & $0 \cdot 2$ & $\mathrm{P}$ \\
\hline 2 & $9 \cdot 1$ & $8 \cdot 7$ & $7 \cdot 7$ & $7 \cdot 5$ & $0 \cdot 1$ & Prot,P \\
\hline \multicolumn{7}{|l|}{ Albumin $(\mathrm{mg} / \mathrm{d})$} \\
\hline Period: 1 & $0 \cdot 58$ & $1 \cdot 10$ & $0 \cdot 86$ & 1.00 & $0 \cdot 16$ & \\
\hline 2 & 0.61 & $1 \cdot 30$ & $0 \cdot 87$ & $1 \cdot 22$ & 0.21 & Prot \\
\hline \multicolumn{7}{|l|}{ Lysozyme (incidence) } \\
\hline Period: 1 & $1 / 6$ & $6 / 6$ & $3 / 6$ & $6 / 6$ & & \\
\hline 2 & $0 / 6$ & $6 / 6$ & $2 / 6$ & $6 / 6$ & & \\
\hline \multicolumn{7}{|l|}{$\begin{array}{l}\text { Urea } \\
\text { (g/kg body-wt per } d)\end{array}$} \\
\hline Period: 1 & $2 \cdot 36$ & $4 \cdot 58$ & $2 \cdot 16$ & $4 \cdot 74$ & $0 \cdot 17$ & Prot \\
\hline 2 & $2 \cdot 26$ & 4.59 & $2 \cdot 13$ & $4 \cdot 50$ & $0 \cdot 19$ & Prot \\
\hline \multicolumn{7}{|l|}{$\begin{array}{l}\text { Creatinine } \\
\text { (mg/kg body-wt per d) }\end{array}$} \\
\hline Period: 1 & $36 \cdot 3$ & $39 \cdot 8$ & $33 \cdot 9$ & $39 \cdot 1$ & $1 \cdot 4$ & Prot \\
\hline 2 & $38 \cdot 5$ & $38 \cdot 5$ & $34 \cdot 7$ & $36 \cdot 1$ & $2 \cdot 0$ & \\
\hline \multicolumn{7}{|c|}{ Plasma variables } \\
\hline Urea (mM) & $6 \cdot 6$ & $8 \cdot 1$ & $6 \cdot 7$ & $8 \cdot 8$ & $0 \cdot 3$ & Prot \\
\hline Creatinine $(\mu \mathrm{M})$ & $30 \cdot 4$ & $34 \cdot 0$ & $30 \cdot 0$ & $31 \cdot 5$ & $2 \cdot 1$ & \\
\hline $\begin{array}{l}\text { Creatinine clearance } \\
\text { (ml/min per } \mathrm{kg} \text { body- } \\
\text { wt) }\end{array}$ & $7 \cdot 9$ & $7 \cdot 0$ & $7 \cdot 1$ & $7 \cdot 2$ & 0.5 & \\
\hline
\end{tabular}

* Dietary variables refer to the calculated amounts of protein, $\mathrm{P}$ and $\mathrm{Mg}$.

$\dagger$ Statistical significance $(P<0.05)$ : Prot, effect of amount of protein; $\mathrm{P}$, effect of amount of $\mathrm{P}$; Prot $\times \mathrm{P}$, effect of interaction.

$\$$ On a $0-3$ scale.

a,b Group means with different superscript letters were significantly different $(P<0.05)$.

\section{Effects of protein intake}

An increase in protein intake, at either low or high dietary $\mathrm{P}$ concentrations, significantly stimulated the excretion of $\mathrm{Ca}$ in urine and tended to increase urinary excretion of $\mathrm{Mg}$ (Table 2). This protein effect was less pronounced if the background diet contained $6 \mathrm{~g} \mathrm{P} / \mathrm{kg}$ instead of $2 \mathrm{~g} \mathrm{P} / \mathrm{kg}$. The high-protein diets significantly lowered the excretion of $\mathrm{Mg}$ in faeces. In Expt 2, an increase in protein intake significantly increased urinary excretion of $\mathrm{Mg}$ (Table 3 ). Urinary $\mathrm{Ca}$ excretion was elevated in rats fed on the high-protein diets only during the first collection period (Table 3 ).

When rats were fed on the high-P diets, containing $6 \mathrm{~g} \mathrm{P} / \mathrm{kg}$ and $0.4 \mathrm{~g} \mathrm{Mg} / \mathrm{kg}$, an increase in dietary protein concentration significantly reduced kidney levels of Ca (Tables 2 and 3). The increase in kidney weight caused by $\mathrm{Mg}$ restriction was counteracted by increasing the protein concentration of the diet (Table 3). In rats fed on the high-Mg diets, an increase in dietary protein tended to increase kidney weight. Nephrocalcinosis induced by decreased $\mathrm{Mg}$ intake, as assessed either chemically or histologically, was nullified by increasing the amount of protein in the diet. 
Table 3. Expt 2. Effects of protein and magnesium intakes on growth and feed intakes, mineral balance and kidney function in rats

(Means with pooled standard errors for eight rats per dictary group)

\begin{tabular}{|c|c|c|c|c|c|c|}
\hline \multirow[b]{2}{*}{$\begin{array}{l}\text { Protein }(\mathrm{g} / \mathrm{kg}) \ldots \\
\operatorname{P}(\mathrm{g} / \mathrm{kg}) \ldots \\
\text { Magnesium }(\mathrm{g} / \mathrm{kg}) \ldots\end{array}$} & \multicolumn{4}{|c|}{ Diet* } & \multirow[b]{2}{*}{ Pooled SE } & \multirow[b]{2}{*}{$\begin{array}{l}\text { Statistical } \\
\text { significance } \dagger\end{array}$} \\
\hline & $\begin{array}{c}151 \\
6 \\
0 \cdot 4\end{array}$ & $\begin{array}{c}302 \\
6 \\
0 \cdot 4\end{array}$ & $\begin{array}{c}151 \\
6 \\
0 \cdot 1\end{array}$ & $\begin{array}{c}302 \\
6 \\
0 \cdot 1\end{array}$ & & \\
\hline \multicolumn{7}{|l|}{ Body-wt (g) } \\
\hline Initial & $164 \cdot 9$ & $164 \cdot 5$ & 1649 & $164 \cdot 1$ & $4 \cdot 7$ & \\
\hline Final & $201 \cdot 7$ & $201 \cdot 6$ & $199 \cdot 5$ & $193 \cdot 5$ & 6.9 & \\
\hline Wt gain $(g / d)$ & $1 \cdot 3$ & $1 \cdot 3$ & $1 \cdot 2$ & $1 \cdot 0$ & $0 \cdot 1$ & $\mathrm{Mg}$ \\
\hline Feed intake $(\mathrm{g} / \mathrm{d})$ & $15 \cdot 4$ & $14 \cdot 7$ & $15 \cdot 0$ & $14 \cdot 3$ & $0 \cdot 5$ & Prot \\
\hline \multicolumn{7}{|c|}{ Mineral balance, period $1(\mathrm{mg} / \mathrm{d})$} \\
\hline Intake & & & & & & \\
\hline Calcium & $61 \cdot 1$ & $61 \cdot 2$ & $61 \cdot 8$ & $56 \cdot 4$ & $2 \cdot 2$ & \\
\hline $\mathrm{Mg}$ & $4 \cdot 9$ & $5 \cdot 1$ & 1.9 & 1.9 & $0 \cdot 2$ & $\mathrm{Mg}$ \\
\hline $\mathbf{P}$ & $79 \cdot 3$ & $73 \cdot 3$ & $74 \cdot 5$ & $63 \cdot 4$ & $2 \cdot 8$ & Prot, $\mathrm{Mg}$ \\
\hline \multicolumn{7}{|l|}{ Urinary output } \\
\hline $\mathrm{Ca}$ & 0.2 & $0 \cdot 3$ & $0 \cdot 1$ & $0 \cdot 2$ & 0.0 & Prot,Mg \\
\hline $\mathrm{Mg}$ & $0 \cdot 4$ & 0.5 & $0 \cdot 1$ & $0 \cdot 3$ & 0.0 & Prot,Mg \\
\hline $\mathrm{P}$ & $32 \cdot 5$ & $33 \cdot 3$ & $34 \cdot 8$ & $35 \cdot 0$ & 1.6 & \\
\hline \multicolumn{7}{|l|}{ Faecal output } \\
\hline $\mathrm{Ca}$ & $43 \cdot 6$ & $42 \cdot 0$ & $37 \cdot 4$ & $39 \cdot 1$ & $2 \cdot 6$ & \\
\hline $\mathrm{Mg}$ & $3 \cdot 6$ & $3 \cdot 2$ & $1 \cdot 5$ & 1.4 & $0 \cdot 2$ & $\mathrm{Mg}$ \\
\hline $\mathbf{P}$ & $26 \cdot 6$ & $24 \cdot 5$ & $23 \cdot 3$ & $21 \cdot 8$ & 1.6 & $\mathrm{Mg}$ \\
\hline \multicolumn{7}{|l|}{ Retention } \\
\hline $\mathrm{Ca}$ & $17 \cdot 3$ & $19 \cdot 0$ & $24 \cdot 3$ & $17 \cdot 1$ & $2 \cdot 3$ & \\
\hline $\mathrm{Mg}$ & 0.9 & $1 \cdot 5$ & $0 \cdot 3$ & $0 \cdot 3$ & $0 \cdot 1$ & Prot,Mg,Prot $\times \mathrm{Mg}$ \\
\hline $\mathbf{P}$ & $20 \cdot 2$ & $15 \cdot 4$ & $16 \cdot 3$ & $8 \cdot 6$ & 1.5 & Prot, $\mathrm{Mg}$ \\
\hline \multicolumn{7}{|c|}{ Mineral balance, period $2(\mathrm{mg} / \mathrm{d})$} \\
\hline Intake & & & & & & \\
\hline $\mathrm{Ca}$ & $63 \cdot 7$ & $62 \cdot 3$ & $63 \cdot 3$ & $61 \cdot 3$ & $2 \cdot 9$ & \\
\hline $\mathrm{Mg}$ & $5 \cdot 1$ & $5 \cdot 2$ & $2 \cdot 0$ & $2 \cdot 1$ & $0 \cdot 2$ & $\mathrm{Mg}$ \\
\hline $\mathbf{P}$ & $82 \cdot 7$ & $74 \cdot 6$ & $76 \cdot 1$ & $71 \cdot 0$ & $3 \cdot 5$ & \\
\hline \multicolumn{7}{|l|}{ Urinary output } \\
\hline $\mathrm{Ca}$ & $0 \cdot 3$ & $0 \cdot 3$ & $0 \cdot 2$ & $0 \cdot 2$ & 0.0 & $\mathrm{Mg}$ \\
\hline $\mathrm{Mg}$ & $0 \cdot 3$ & 0.6 & $0 \cdot 1$ & $0 \cdot \overline{2}$ & 0.0 & Prot, Mg \\
\hline $\mathbf{P}$ & $31 \cdot 8$ & $32 \cdot 3$ & $34 \cdot 1$ & $33 \cdot 2$ & $1 \cdot 7$ & \\
\hline \multicolumn{7}{|l|}{ Faecal output } \\
\hline $\mathrm{Ca}$ & $45 \cdot 8$ & $41 \cdot 2$ & $40 \cdot 8$ & $40 \cdot 3$ & $2 \cdot 4$ & \\
\hline $\mathrm{Mg}$ & $3 \cdot 9$ & $3 \cdot 2$ & 1.6 & 1.5 & $0 \cdot 2$ & Prot, $\mathrm{Mg}$ \\
\hline $\mathrm{P}$ & $29 \cdot 0$ & $24 \cdot 9$ & $25 \cdot 3$ & $23 \cdot 7$ & $1 \cdot 6$ & \\
\hline \multicolumn{7}{|l|}{ Retention } \\
\hline $\mathrm{Ca}$ & $18 \cdot 3$ & $20 \cdot 8$ & $22 \cdot 3$ & $20 \cdot 8$ & $2 \cdot 1$ & \\
\hline $\mathrm{Mg}$ & 0.9 & 1.5 & $0 \cdot 3$ & 0.4 & $0 \cdot 1$ & Prot,Mg \\
\hline $\mathrm{P}$ & $22 \cdot 0$ & $17 \cdot 4$ & $16 \cdot 8$ & $14 \cdot 1$ & 1.8 & $\mathrm{Mg}$ \\
\hline \multicolumn{7}{|c|}{ Kidney variables } \\
\hline $\begin{array}{l}\text { Relative kidney wt } \\
\text { (g/kg body-wt) }\end{array}$ & $3 \cdot 7$ & $4 \cdot 0$ & $4 \cdot 7$ & $4 \cdot 3$ & $0 \cdot 2$ & Mg,Prot $\times$ Mg \\
\hline Dry wt $(\mathrm{mg})$ & 166 & 175 & 188 & 179 & 7 & \\
\hline $\mathrm{Ca}(\mathrm{g} / \mathrm{kg})$ & 1.05 & $0 \cdot 50$ & $20 \cdot 65$ & 0.71 & 0.25 & Prot, $\mathrm{Mg}$, Prot $\times \mathrm{Mg}$ \\
\hline $\mathrm{Mg}(\mathrm{g} / \mathrm{kg})$ & 0.83 & 0.86 & 0.98 & $0 \cdot 81$ & $0 \cdot 01$ & Prot, Prot $\times \mathrm{Mg}$ \\
\hline$P(g / k g)$ & $12 \cdot 6$ & $12 \cdot 4$ & $24 \cdot 2$ & $12 \cdot 4$ & $0 \cdot 1$ & Prot, $\mathrm{Mg}$, Prot $\times \mathrm{Mg}$ \\
\hline \multicolumn{7}{|l|}{ Nephrocalcinosis } \\
\hline Incidence & $3 / 8$ & $1 / 8$ & $8 / 8$ & $1 / 8$ & & \\
\hline Severity: & $0.4^{b}$ & $0 \cdot 0^{\mathrm{a}}$ & $2 \cdot 6^{\mathrm{c}}$ & $0 \cdot 0^{\mathrm{a}}$ & & \\
\hline
\end{tabular}


Table 3. (cont.)

\begin{tabular}{|c|c|c|c|c|c|c|}
\hline \multirow{4}{*}{$\begin{array}{l}\text { Protein }(\mathrm{g} / \mathrm{kg}) \ldots \\
\mathrm{P}(\mathrm{g} / \mathrm{kg}) \ldots \\
\text { Magnesium }(\mathrm{g} / \mathrm{kg}) \ldots\end{array}$} & \multicolumn{4}{|c|}{ Diet* } & \multirow[b]{4}{*}{ Pooled SE } & \multirow{4}{*}{$\begin{array}{l}\text { Statistical } \\
\text { significancet }\end{array}$} \\
\hline & 151 & 302 & 151 & 302 & & \\
\hline & 6 & 6 & 6 & 6 & & \\
\hline & 0.4 & $0 \cdot 4$ & $0 \cdot 1$ & $0 \cdot 1$ & & \\
\hline \multicolumn{7}{|c|}{ Urinary output } \\
\hline \multicolumn{7}{|l|}{ Production (ml/d) } \\
\hline Period: 1 & $15 \cdot 0$ & $10 \cdot 8$ & $15 \cdot 3$ & $16 \cdot 2$ & $8 \cdot 3$ & \\
\hline 2 & $10 \cdot 9$ & $10 \cdot 7$ & $14 \cdot 2$ & $15 \cdot 4$ & $5 \cdot 6$ & $\mathrm{Mg}$ \\
\hline \multicolumn{7}{|l|}{$\mathrm{pH}$} \\
\hline Period: 1 & $8 \cdot 0$ & $7 \cdot 5$ & $7 \cdot 6$ & $7 \cdot 7$ & $0 \cdot 1$ & Prot, Prot $\times \mathrm{Mg}$ \\
\hline 2 & $7 \cdot 8$ & $7 \cdot 5$ & $7 \cdot 6$ & $7 \cdot 8$ & $0 \cdot 1$ & Prot $\times \mathrm{Mg}$ \\
\hline \multicolumn{7}{|l|}{ Albumin $(\mathrm{mg} / \mathrm{d})$} \\
\hline Period: 1 & 0.48 & $0 \cdot 87$ & 0.94 & 0.78 & $0 \cdot 25$ & \\
\hline 2 & $0 \cdot 77$ & 1.08 & 0.92 & $0 \cdot 94$ & $0 \cdot 32$ & \\
\hline \multicolumn{7}{|l|}{ Lysozyme (incidence) } \\
\hline Period: 1 & $0 / 8$ & $2 / 8$ & $0 / 8$ & $1 / 8$ & & \\
\hline 2 & $2 / 8$ & $3 / 8$ & $0 / 8$ & $2 / 8$ & & \\
\hline \multicolumn{7}{|l|}{$\begin{array}{l}\text { Urea } \\
(\mathrm{g} / \mathrm{kg} \text { body-wt per } \mathrm{d})\end{array}$} \\
\hline Period: 1 & $2 \cdot 13$ & $4 \cdot 44$ & $2 \cdot 14$ & $4 \cdot 53$ & $0 \cdot 12$ & Prot \\
\hline 2 & $2 \cdot 09$ & $4 \cdot 33$ & $2 \cdot 14$ & $4 \cdot 34$ & $0 \cdot 14$ & Prot \\
\hline \multicolumn{7}{|l|}{$\begin{array}{l}\text { Creatinine } \\
(\mathrm{mg} / \mathrm{kg} \text { body-wt per } \mathrm{d})\end{array}$} \\
\hline Period: 1 & $39 \cdot 1$ & $40 \cdot 2$ & $39 \cdot 1$ & $41 \cdot 3$ & $1 \cdot 6$ & \\
\hline 2 & $33 \cdot 8$ & $37 \cdot 2$ & $37 \cdot 4$ & $37 \cdot 5$ & $1 \cdot 4$ & \\
\hline \multicolumn{7}{|c|}{ Plasma variables } \\
\hline Urea $(\mathrm{mm})$ & $5 \cdot 7$ & $7 \cdot 8$ & $7 \cdot 4$ & $8 \cdot 2$ & $0 \cdot 3$ & Prot,Mg \\
\hline Creatinine $(\mu \mathrm{M})$ & $35 \cdot 9$ & $38 \cdot 9$ & $36 \cdot 8$ & $32 \cdot 0$ & $1 \cdot 8$ & Prot $\times \mathrm{Mg}$ \\
\hline $\begin{array}{l}\text { Creatinine clearance } \\
(\mathrm{ml} / \mathrm{min} \text { per } \mathrm{kg} \text { body- } \\
\text { wt) }\end{array}$ & $5 \cdot 8$ & $6 \cdot 1$ & $6 \cdot 3$ & $7 \cdot 4$ & 0.6 & \\
\hline
\end{tabular}

* Dietary variables refer to the calculated amounts of protein, $\mathrm{P}$ and $\mathrm{Mg}$.

$\dagger$ Statistical significance $(P<0.05)$ : Prot, effect of amount of protein; $\mathrm{Mg}$, effect of amount of $\mathrm{Mg}$; Prot $\times \mathrm{Mg}$, effect of interaction.

$\ddagger$ On a $0-3$ scale.

at, b, "Group means with different superscript letters were significantly different $(P<0.05)$.

An increase in protein intake with low- or high-P diets containing $0.4 \mathrm{~g} \mathrm{Mg} / \mathrm{kg}$ caused enhanced albumin loss in urine, but this effect did not reach statistical significance (Tables 2 and 3). The incidence of lysozyme-positive urine was on average higher in rats fed on the high-protein diets compared with low-protein diets. Increasing the concentration of protein in the diet significantly increased urea output in urine and its concentration in plasma. Urinary creatinine and creatinine clearance were not significantly influenced by the amount of protein in the diet.

\section{DISCUSSION}

An increase in the concentration of $\mathrm{P}$ in the diet from 2 to $6 \mathrm{~g} / \mathrm{kg}$ or a decrease in $\mathrm{Mg}$ concentration from 0.4 to $0.1 \mathrm{~g} / \mathrm{kg}$, with a dietary background of $151 \mathrm{~g}$ protein $/ \mathrm{kg}$ diet, caused nephrocalcinosis as based on increased $\mathrm{Ca}$ concentrations in kidney and the formation of Von Kossa-positive material in kidney sections. In rats fed on similar diets, the degree of nephrocalcinosis differed markedly in Expts 1 and 2. There was a 10-fold difference between experiments in kidney $\mathrm{Ca}$ in rats fed on the diet with $151 \mathrm{~g}$ protein $/ \mathrm{kg}$ 
containing $6 \mathrm{~g} \mathrm{P} / \mathrm{kg}$ and $0.4 \mathrm{~g} \mathrm{Mg} / \mathrm{kg}$ (Tables 2 and 3). In our earlier studies (Hoek et al. 1988; Mars et al. 1988; Ritskes-Hoitinga et al. 1989) it was also found that in rats fed on nephrocalcinogenic diets the absolute concentrations of kidney $\mathrm{Ca}$ are poorly reproducible. However, dietary effects within experiments have proved quite reproducible. Likewise, in the present study the anti-nephrocalcinogenic effect of high protein intake was seen in rats fed on diets containing $6 \mathrm{~g} \mathrm{P} / \mathrm{kg}$ and $0.4 \mathrm{~g} \mathrm{Mg} / \mathrm{kg}$ both in Expt 1 (Table 2) and Expt 2 (Table 3). It would appear that fluctuations in intangible factors determine the absolute rather than relative sensitivity of rats to nephrocalcinogenic diets. Thus, it can be concluded from the present study that an increase in dietary protein concentration from 151 to $302 \mathrm{~g} / \mathrm{kg}$ completely counteracted nephrocalcinogenesis induced by either increased $\mathrm{P}$ or decreased $\mathrm{Mg}$ intake. It is unlikely that this protein effect refers to a specific characteristic of ovalbumin. There is evidence that increased intakes of casein and soya-bean protein also reduce nephrocalcinosis (Eklund et al. 1973; Kaunitz \& Johnson, 1976; Schneider \& Menden, 1988), but the diets used were not balanced for minerals in the proteins.

One objective of the present study was to determine whether the inhibitory effect of high protein intake on nephrocalcinosis is related to protein-induced magnesiuria. In order to reduce the risk of finding a spurious relationship, nephrocalcinosis was induced by two different dietary changes, that is by P loading in Expt 1 and by $\mathrm{Mg}$ restriction in Expt 2 . The protein-induced inhibition of nephrocalcinogenesis was paralleled by increased urinary excretion of $\mathrm{Mg}$ in Expts 1 and 2. Only in the first balance period of Expt 1 did the protein effect not reach statistical significance. Thus, the present study would support a cause-andeffect relationship between nephrocalcinogenesis and urinary $\mathrm{Mg}$ excretion, if any. Other present observations speak against such a causal relationship. In keeping with earlier studies (Hoek et al. 1988; Mars et al. 1988), an increase in P intake in rats fed on a diet containing $151 \mathrm{~g}$ protein $/ \mathrm{kg}$ lowered $\mathrm{Mg}$ excretion in urine. This $\mathrm{P}$ effect was of the same order of magnitude in rats fed on a diet containing $302 \mathrm{~g}$ protein $/ \mathrm{kg}$, but the degree of nephrocalcinosis was not influenced in this situation (Table 2). The same conclusion would be reached when urinary $\mathrm{Mg}$ concentrations are considered (cf. Table 2). A decrease in $\mathrm{Mg}$ intake by rats fed on the low-protein diet resulted in marked nephrocalcinosis associated with a decrease in $\mathrm{Mg}$ excretion in urine (Table 3). Lowering of the $\mathrm{Mg}$ concentration in the high-protein diet reduced urinary $\mathrm{Mg}$ excretion but did not produce nephrocalcinosis. Thus, urinary $\mathrm{Mg}$ concentrations and nephrocalcinosis appear not to be proportionally related. It is also questionable whether the two variables are causally associated.

With diets containing $151 \mathrm{~g}$ protein $/ \mathrm{kg}$, an increase in $\mathrm{P}$ intake or decrease in $\mathrm{Mg}$ intake resulted in increased group mean urinary excretion of albumin (Tables 2 and 3). As suggested earlier (Ritskes-Hoitinga et al. 1989), this could be related to nephrocalcinosisinduced kidney damage. Albumin excretion is assumed to reflect glomerular leakage or defective re-absorption in the proximal tubules, or both (Massry \& Glassock, 1983). Massive nephrocalcinosis induced by decreased $\mathrm{Mg}$ intake with the diet containing $151 \mathrm{~g}$ protein $/ \mathrm{kg}$ was associated with increased group mean plasma concentrations of urea (Table 3). This would also point to kidney damage as a result of nephrocalcinosis. In keeping with the proposition that nephrocalcinosis causes kidney damage, Kang et al. (1979) found increased urinary $N$-acetyl- $\beta$-glucosaminidase (EC 3.2.1.30) activity in rats with nephrocalcinosis.

Since high protein intakes reduce nephrocalcinosis, it would be anticipated that high protein intakes lower albumin excretion in urine. However, such an effect was not found. High protein intake itself (Expt 1) might cause kidney damage, resulting in increased albumin excretion (Rosenberg et al. 1988). This would be supported by the observed protein-induced increase of urinary excretion of lysozyme in Expt 1 . However, urinary lysozyme could not be detected in Expt 2. Lysozyme excretion points to damage of the 
proximal tubulus (Balazs \& Roepke, 1966; Harrison et al. 1973). The protein-induced increased urinary urea excretion and increased concentrations of urea in plasma are most likely related to enhanced protein catabolism rather than kidney damage (Rosenberg et al. 1988). There was no systematic effect on creatinine clearance, implying that the glomerular filtration rate was not influenced by feeding the high-protein diets. In contrast, Bouby et al (1988) found a significant increase in creatinine clearance, glomerular filtration rate and kidney mass in male rats after feeding for 6 weeks a diet containing $320 \mathrm{~g}$ casein $/ \mathrm{kg}$, compared with a diet containing $100 \mathrm{~g}$ casein $/ \mathrm{kg}$. The diets were balanced for $\mathrm{P}$ content, but not for $\mathrm{Ca}$ and $\mathrm{Mg}$. The fact that we used rats of another sex and strain and a shorter feeding period may explain the discrepancy between the outcome of the study of Bouby et al. (1988) and that of the present study. Stonard et al. (1984) did not measure a different inulin clearance after feeding two protein levels $(300 \mathrm{v} .180 \mathrm{~g} / \mathrm{kg})$. Thus, the amount of protein in the diet may not consistently alter the glomerular filtration rate in rats.

In the present study, the addition of extra protein to the non-nephrocalcinogenic basal diets caused an increased relative kidney weight. This is in accordance with the findings of Bouby et al. (1988). Nephrocalcinosis also caused an increased kidney weight which is due not only to the Ca deposits, but also to hypertrophy (Ritskes-Hoitinga et al. 1989). The addition of protein to nephrocalcinogenic diets did not clearly affect kidney weight. This suggests that under this condition the protein-induced increase in kidney weight and the decrease in kidney weight associated with diminished nephrocalcinosis are of the same order of magnitude. The hypertrophy on high-protein diets is characterized by a preferential increase in the thickness of the inner stripe of the outer medulla, coinciding with marked hypertrophy of the thick ascending limb of Henle's loop (Bouby et al. 1988). Possibly the hypertrophy of the thick ascending limb on a high-protein diet relates to decreased sensitivity towards the development of kidney calcification.

In summary, increased intake of protein very effectively counteracted nephrocalcinogenesis in female rats induced by the feeding of either high-P or low-Mg diets. The metabolic basis for this protein effect is not yet clear. Further work on this issue is relevant as it will broaden our basic knowledge of interactions between diet composition and nephrocalcinogenesis, which can occur in various animal species, including man. Ultimately, this knowledge may assist in treating or preventing kidney calcification, or both.

The authors thank J. W. M. Haas and G. van Tintelen (Laboratory Animals Centre, Agricultural University, Wageningen) for taking care of the rats.

\section{REFERENCES}

Al-Modhefer, A. K. J., Atherton, J. C., Garland, H. O., Singh, H. J. \& Walker, J. (1986). Kidney function in rats with corticomedullary nephrocalcinosis: effects of alterations in dietary calcium and magnesium. Journal of Physiology 380, 405-414.

Balazs, T. \& Roepke, R. R. (1966). Lysozymuria induced in rats by nephrotoxic agents. Proceedings of the Society for Experimental Biology and Medicine 123, 380 385.

Bouby, N., Trinh-Trang-Tan, M. M., Laouari, D., Kleinknecht, C., Grünfeld, J. P., Kriz, W. \& Bankir, L. (1988). Role of the urinary concentrating process in the renal effects of high protein intake. Kidney International 34, 4-12.

Boulet, M., Marier, J. R. \& Rose, D. (1962). Effect of magnesium on formation of calcium phosphate precipitates. Archives of Biochemistry and Biophysics 96, 629-636.

Bunce, G. E. \& Bloomer, J. E. (1972). Effect of magnesium deficiency on serum and urinary ions in rats: studies with ion-selective electrodes. Journal of Nutrition 102, 863-872.

Bunce, G. E., Sauberlich, H. E., Reeves, P. G. \& Oba, T. S. (1965). Dietary phosphorus and magnesium deficiency in the rat. Journal of Nutrition 86, 406-414.

Draper, H. H., Sie, T. L. \& Bergan, J. G. (1972). Osteoporosis in aging rats induced by high phosphorus diets. Journal of Nutrition 102, 1133-1142. 
Du Bruyn, D. B. (1972). Nephrocalcinosis in the white rat. Part 2. The relationship between dietary magnesium, calcium and phosphorus content and kidney calcification and bone magnesium. South African Medical Journal 46, $1588-1593$.

Eklund, A, Agren, G., Nordgren, H. \& Stenram, U. (1973). Nephrocalcinosis in adolescent Sprague-Dawley rats fed casein and different salt mixtures. Nutrition and Metabolism 15, 348-356.

Goulding, A. \& Malthus, R. S. (1969). Effect of dietary magnesium on the development of nephrocalcinosis in rats. Journal of Nutrition $97,353-358$.

Harrison, J. F., Parker, R. W. \& De Silva, K. L. (1973). Lysozymuria and acute disorders of renal function. Journal of Clinical Pathology 26, 278-284.

Harwood, E. J. (1982). The influence of dietary magnesium on reduction of nephrocalcinosis in rats fed purified diets. Laboratory Animals 16, 314-318.

Hellerstein, E., E., Vitale, J. J., White, P. L., Hegsted, D. M., Zamcheck, N. \& Nakamura, M. (1957). Influence of dietary magnesium on cardiac and renal lesions of young rats fed an atherogenic diet. Joumal of Experimentat Medicine 106, 767-776.

Hitchman, A. J., Hasany, S. A., Hitchman, A., Harrison, J. E. \& Tam, C. (1979). Phosphate-induced renal calcification in the rat. Canadian Journal of Physiology and Pharmacology 57, 92-97.

Hoek, A. C., Lemmens, A. G., Mullink, J. W. M. A. \& Beynen, A. C. (1988). Influence of dietary calcium:phosphorus ratio on mineral excretion and nephrocalcinosis in female rats. Journal of Nurition 118 $1210-1216$.

Kang, S. S., Price, R. G., Yudkin, J., Worcester, N. A. \& Bruckdorfer, K. R. (1979). The influence of dietary carbohydrate and fat on kidney calcification and the urinary excretion of $N$-acetyl- $\beta$-glucosaminidase. British Journal of Nutrition 41, 65-71.

Kaunitz, H. \& Johnson, R. E. (1976). Dietary protein, fat and minerals in nephrocalcinosis in female rats. Metabolism 25, 69-77.

Mallory, F. B. (1961). Pathological Technique. New York: Hafner Publishing Co.

Mancini, G., Carbonara, A. O. \& Heremans, J. F. (1965). Immunochemical quantitation of antigens by single radial immunodiffusion. Immunochemistry 2, 235-239.

Mars, Y. W. H. M., Lemmens, A. G. \& Beynen, A. C. (1988). Dietary phosphorus and nephrocalcinosis in female rats. Nutrition Reports International 38, 249-258.

Massry, S. G. \& Glassock, R. J. (1983). Textbook of Nephrology. Baltimore: Williams and Wilkins.

Osserman, E. F. \& Lawlor, D. P. (1966). Serum and urinary lysozyme (muramidase) in monocytic and monomyelocytic leukemia. Journal of Experimental Medicine 124, 921-951.

Parker, H. E. (1985). Magnesium and phosphorus interrelationships at deficient to normal dietary levels. Nuirition Reports International 32, 983-990

Ritskes-Hoitinga, J., Lemmens, A. G. \& Beynen, A. C. (1989). Nutrition and kidney calcification in rats. Laboratory Animals 23, 313-318.

Rosenberg, M. E., Swanson, J. E., Thomas, B. L., Chmielewski, D. \& Hostetter, T. H. (1988). Dietary protein restriction lessens the glomerular permselective defect in human renal disease. Contributions to Nephrology $\mathbf{6 8}$, $157-165$.

Schneider, W. \& Menden, E. (1988). Der Einflu $\beta$ langfristig überhöhter Proteinzufuhr auf der Mineralstoffwechsel und die Nierenfunktion der Ratte. I. Die renale und enterale Ausscheidung von Calcium, Magnesium, Phosphor, Sulfat und Säure. (The long-term influence of increased protein supply on mineral metabolism and kidney function in the rat. I. Renal and intestinal elimination of calcium, magnesium, phosphorus, sulphate and acids.) Zeitschrift für Ernährungswissenschaft 27, 170-185.

Stonard, M. D., Samuels, D. M. \& Lock, E. A. (1984). The pathogenesis and effect on renal function of nephrocalcinosis induced by different diets in female rats. Food and Chemical Toxicology 22, 139-146.

Taussky, H. H. \& Shorr, E. (1953). A microcolorimetric method for the determination of inorganic phosphorus Journat of Biological Chemistry 202, 675-685. 